\title{
Improvement in design of noise shaping filters
}

Research article

\author{
Dejan G. Ćirić ${ }^{1}$, Miloš Markovićc ${ }^{1,2 *}$, Branko Stojić ${ }^{1}$ \\ 1 Faculty of Electronic Engineering, University of Niš, Aleksandra Medvedeva 14, 18000 Niš, Serbia \\ 2 Currently at: Section of Acoustics, Department of Electronic Systems, Aalborg University, \\ Fredrik Bajers Vej 7 B5, 9220 Aalborg Ø, Denmark
}

Received 07 October 2011 ; accepted 10 February 2012

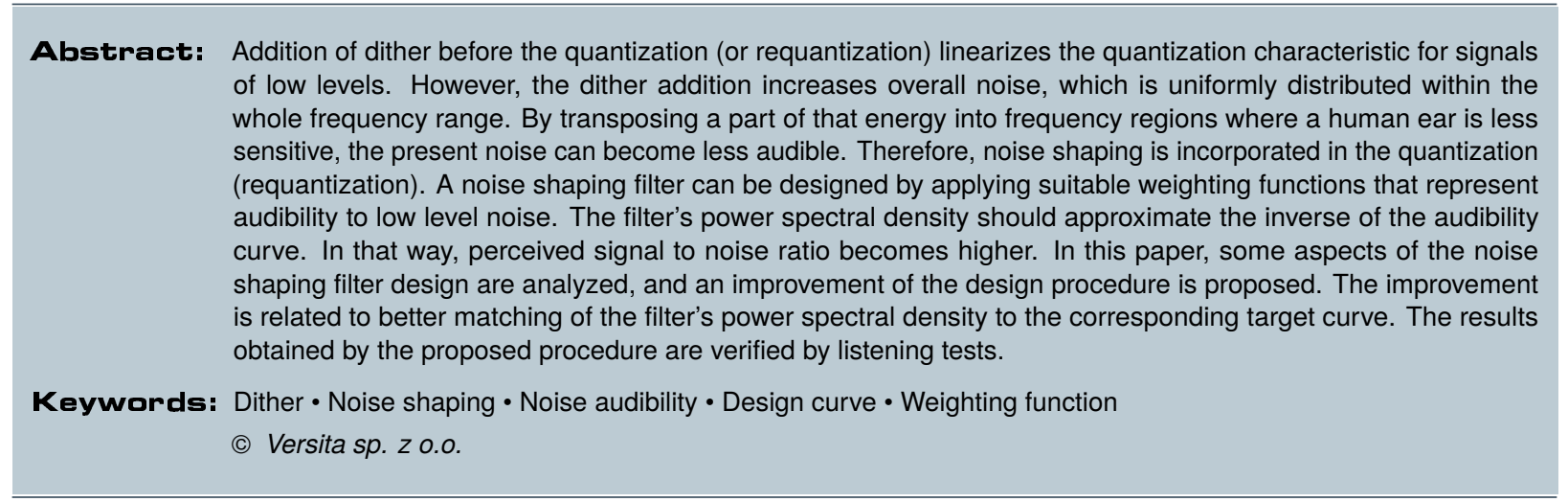

\section{Introduction}

One of the necessary steps in analogue to digital signal conversion is quantization, which sometimes includes requantization, as well. An integral part of that process is a dither addition and noise shaping [1]. The dither addition improves quantized signal resolution at low signal levels and enables reduction of the distortion components [2-4]. However, simple dither addition (non-subtractive dithering) causes an increase in overall noise, which is uniformly distributed within the whole frequency range [4] Nevertheless, such a signal is perceptually less disturbing than higher harmonic component distortion [1, 3-5]. One

*E-mail: mio@es.aau.dk of the ways to reduce the dither contribution to overall noise is to use a dither subtraction after the quantization $[1,3]$. On the other hand, regardless of dithering, a certain amount of output noise, which is generally white, is always present.

To achieve a further improvement in reducing the perceived noise, another approach is used. Audibility of the increased noise can be minimized by transposing a part of the noise energy into frequency regions where a human ear is less sensitive [6-8]. For that purpose, noise shaping is incorporated in the requantization process using a noise shaping filter. In the requantization topology, the quantizer output error is fed back through the noise shaping filter where it is spectrally shaped, and then subtracted from the quantizer input (see, e.g., $[1,3,6])$. In order to achieve the mentioned transposing, the filter's power spec- 
tral density (PSD) should match the corresponding design curve (weighting function) as closely as possible. During the previous period, several weighting functions have been implemented in the noise shaping filter design $[6,7,9,10]$. Besides, various procedures have been used for designing noise shaping filters $[6,7,11-13]$.

Noise shaping filters obtained by some of the design procedures have PSD significantly different from the aimed design curve (target curve). The difference is especially prominent in the regions of the ear's greatest sensitivity $[9,10]$. To overcome this problem, an alternative method of the design is proposed in this paper. This is carried out through the corresponding procedure for both adaptive and fixed filter design [2]. For the sake of comparison, another procedure presented in the literature [7] is also applied. Two weighting functions, called modified $E[6]$ and modified $F$ [7] weighting functions, as well as their modified versions proposed here are used for the filter design. The improvement in shaped noise audibility enabled by the proposed modified weighting functions (design procedure) is examined through the listening tests.

\section{Noise shaping filter design}

A noise shaping filter is designed using a weighting function that should accurately represent the ear's sensitivity to low level broadband noise as a function of frequency. In that regard, audibility of the shaped noise strongly depends on the weighting function. There is no weighting function adopted as a standardized function. As a consequence, several functions have been used for this purpose including $E$ weighting function and its modifications (such as modified $E$ and improved $E$ function [6]), than so called $F$ function and modified $F$ function [7], etc. The modified $E$, original $F$ and modified $F$ weighting functions are shown in Fig. 1. Both the modified $E$ and modified $F$ functions are used in this paper as weighting functions. The curves applied for noise shaping filter design (design curves) are obtained from the mentioned functions by inversion and normalization in such a way that their average logarithmic PSDs are equal to zero.

\subsection{Procedures for noise shaping filter design}

The aim of a noise shaping filter design procedure is to design a filter so that its PSD matches the corresponding design curve as closely as possible. The filter, $H(z)$, obtained in this way minimizes the total perceptually weighted output noise, $N_{\omega}$, as much as possible:

$$
N_{\omega}=\frac{1}{\omega_{N}} \int_{0}^{\omega_{N}}|1-H(z)|^{2} W(\omega) d \omega
$$

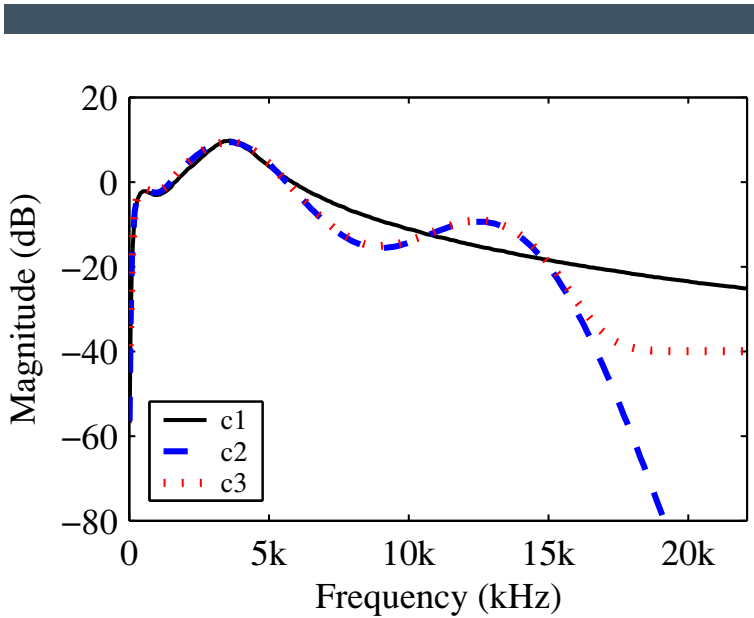

Figure 1. The weighting functions: modified $E$ (c1), original $F$ (c2) and modified $F$ (c3) function.

where $W(\omega)$ is the perceptual weighting function, $\omega=2 \pi f$ ( $f$ is the frequency), and $\omega_{N}=2 \pi\left(f_{s} / 2\right)$, where $f_{s}$ is the sampling frequency whose value is typically $44.1 \mathrm{kHz}$.

This paper deals with two design procedures already presented in the literature [2, 7], where only finite impulse response (FIR) filters are designed. The transfer function of these filters is:

$$
H(z)=z^{-1}\left(a_{0}+a_{1} z^{-1}+a_{2} z^{-2}+\ldots\right) .
$$

The leading delay of one sample $z^{-1}$ is inherent in the mentioned requantization topology because the error is not obtained before carrying out the quantization process.

\subsubsection{Design procedure I}

The first design procedure used in this paper is based on the optimization of filter coefficients in such a manner that $H_{n}(z)=1-H(z)$ matches the design curve $S(\omega)$ as closely as possible, where $z=e^{-j \omega t}$. This procedure differs from some others in that $S(\omega)$ represents the desired noise spectrum instead of masked error PSD [7].

If Equation 1 is rewritten to take a suitable form:

$$
T(a)=\frac{1}{\omega_{N}} \int_{0}^{\omega_{N}} \frac{1}{S(\omega)}\left|H_{n}\left(e^{-j \omega t}\right)\right|^{2} d \omega,
$$

then the optimization is performed by calculation of:

$$
\frac{\partial T(a)}{\partial a_{i}}=0, \quad i \in\{0, \ldots, q-1\},
$$

where $q$ is the number of filter coefficients. Equation 3 is minimal only if $H_{n}\left(e^{-j \omega t}\right)$ is a minimum-phase filter. More details on this procedure can be found in [2].

The described procedure is applied here by the module developed in Matlab for designing FIR filters of various orders using both design curves (modified $E$ and modified $F$ ). 
Table 1. RMS errors (deviations) between the PSDs of the filters designed by the procedure I and II using the corresponding design curves (modified $E$, modified $F$, new modified $E$ and new modified $F$ ) and the target curves (modified $E$ and modified $F$ )

\begin{tabular}{ccccccc}
\hline \multirow{2}{*}{$\begin{array}{c}\text { filter } \\
\text { order }\end{array}$} & $\begin{array}{c}\text { proc. I, } \\
\text { mod. } E\end{array}$ & $\begin{array}{c}\text { proc. I, } \\
\text { mod. } F\end{array}$ & $\begin{array}{c}\text { proc. II, } \\
\text { mod. } E\end{array}$ & $\begin{array}{c}\text { proc. II, } \\
\text { mod. } F\end{array}$ & $\begin{array}{c}\text { proc. I, } \\
\text { new mod. } E\end{array}$ & $\begin{array}{c}\text { proc. I, } \\
\text { new mod. } F\end{array}$ \\
\hline \hline 2 & 5.54 & 11.13 & 3.62 & 11.86 & 2.53 & 9.07 \\
\hline 5 & 5.40 & 8.51 & 2.53 & 8.65 & 2.06 & 5.38 \\
\hline 9 & 8.35 & & & 1.55 & 2.91 \\
\hline 15 & 5.34 & 8.33 & & & \\
\hline 25 & 7.59 & & \\
\hline
\end{tabular}
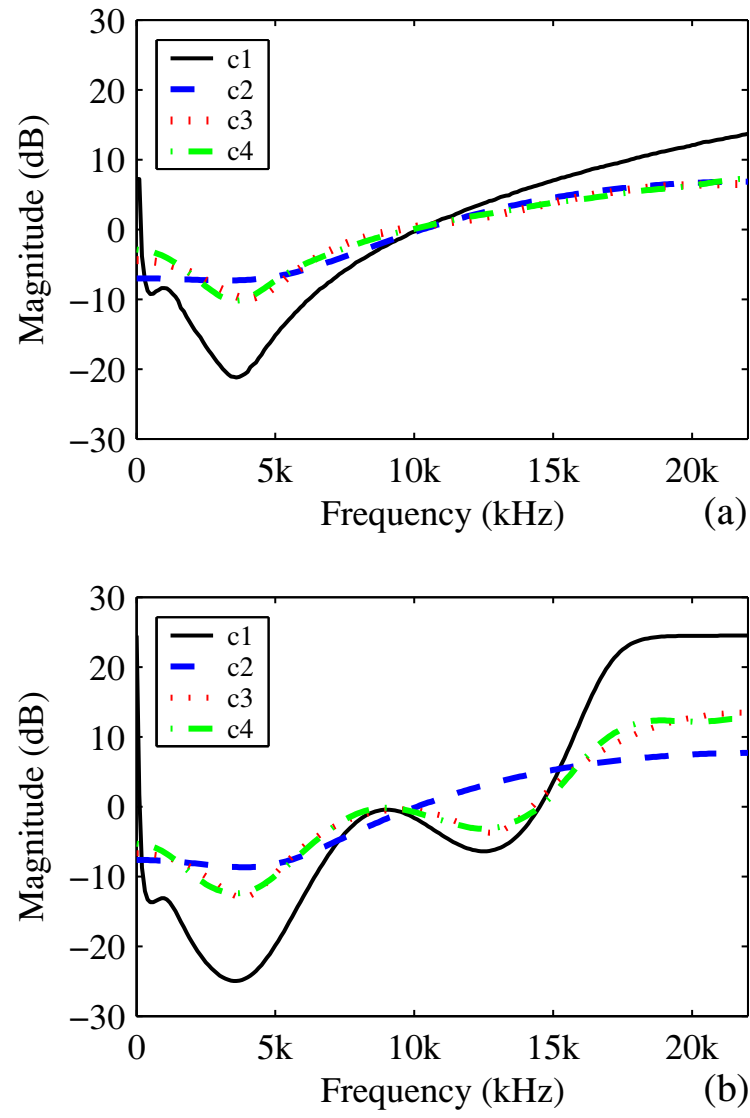

Figure 2. The design curves (c1) - modified $E(a)$ and modified $F$ (b) together with PSDs of the filters of $2^{\text {nd }}(c 2), 5^{\text {th }}$ (c3) and $15^{\text {th }}$ (c4) order designed by the procedure I.

The PSDs of the designed noise shaping filters are shown in Fig. 2.

In order to quantify the difference between the filters' PSDs and the design (target) curves, the root mean squared (RMS) errors are calculated for all filters whose PSDs are presented in this paper, and they are summarized in Table 1.

\subsubsection{Design procedure II}

In the second design procedure, a property that the inverse fast Fourier transform (IFFT) of completely specified characteristic $1-H\left(e^{-j \omega t}\right)$ gives the impulse response of corresponding FIR filter is used [7]. In this case, the design curve represents the squared magnitude of the frequency response of a minimum-phase filter $\left|1-H\left(e^{-j \omega t}\right)\right|^{2}$. The phase response can be obtained by implementation of the Hilbert transform [7].

This procedure (procedure II) is also applied here by another module developed in Matlab for designing another set of noise shaping filters of various orders using two previously mentioned design curves. The designed filters' PSDs are presented in Fig. 3. These filters show good characteristics, but only if the number of filter coefficients is greater than 20. This is because the amplitude of the filter impulse response is typically small only after 20 samples.

\section{Improvement in noise shaping fil- ter design}

In order to obtain the filters whose PSDs deviate less from the design curves in the frequency regions of the human ear's greatest sensitivity, a modification of the design procedure I is proposed in this paper. It is achieved through a modification of the design curves. This approach is chosen because of low computational requirements and the fact that it enables designing the filters of low orders with good characteristics.

To reduce the difference between the filter's PSD and corresponding target curve, the design curve is modified in a specific way (it is made to be different from the target curve). Thus, the dips in the regions of the ears' greatest sensitivity are made to be bigger, while the PSD magnitude is increased at higher frequencies. The design curve should have bigger dips in each next step of design in the mentioned regions until the difference between the filter's 

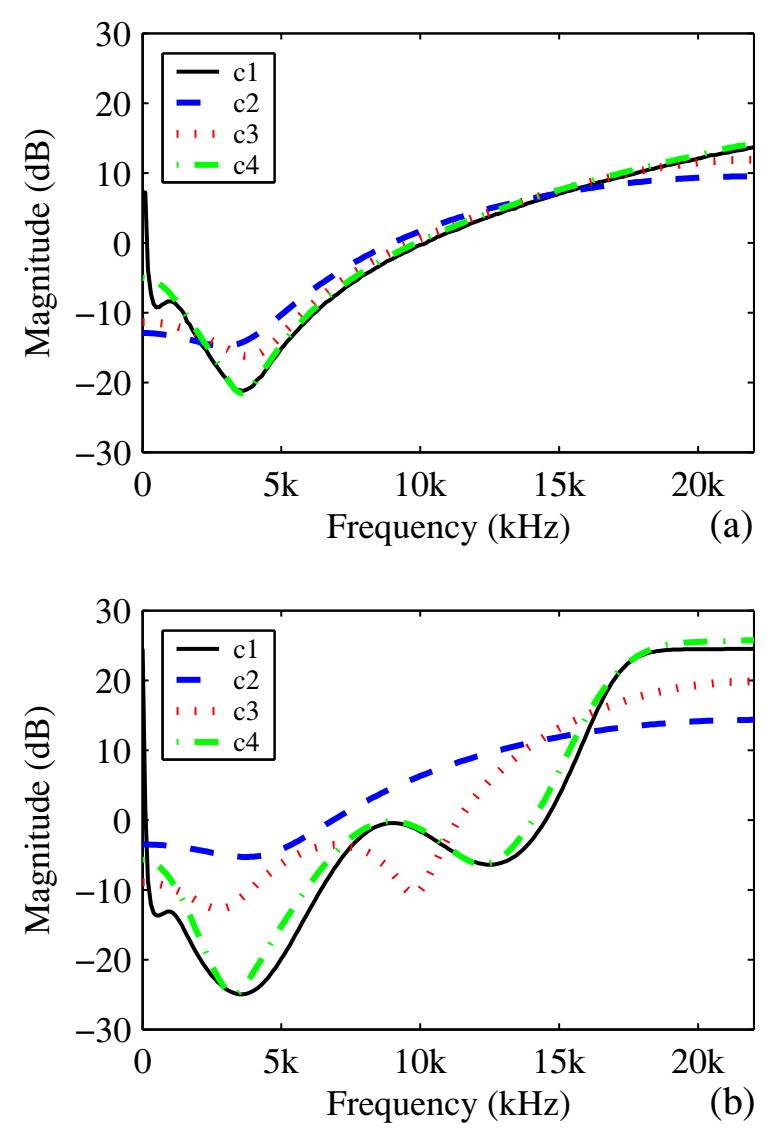

Figure 3. The design curves (c1) - modified $E$ (a) and modified $F$ (b) together with PSDs of the filters of $2^{\text {nd }}(\mathrm{c} 2), 5^{\text {th }}$ (c3) and $25^{\text {th }}(\mathrm{c} 4)$ order designed by the procedure II.

PSD and target curve becomes acceptable (e.g. smaller than the defined threshold). In this way, the design procedure becomes iterative. The desired filter's PSD can be obtained in only a few steps. Alternatively, using the appropriately modified design curve, the design procedure can be non-iterative (done in one step).

The design curves used in the previous examples (modified $E$ and modified $F$ ) together with the versions of these curves modified as explained above (referred to as new modified $E$ and new modified $F$ ) are shown in Fig. 4. These new (modified) curves are also normalized so that their average logarithmic PSDs are equal to zero.

The modified design procedure is applied in a similar way as the procedures I and II. The results of its application (the filters' PSDs) when the new modified $E$ and new modified $F$ curves are used as design curves are presented in Fig. 5(a) and 5(b), respectively. The improvement in noise shaping filter design enabled by the proposed modification is illustrated by the example of the filter of order 9 shown in Fig. 6.
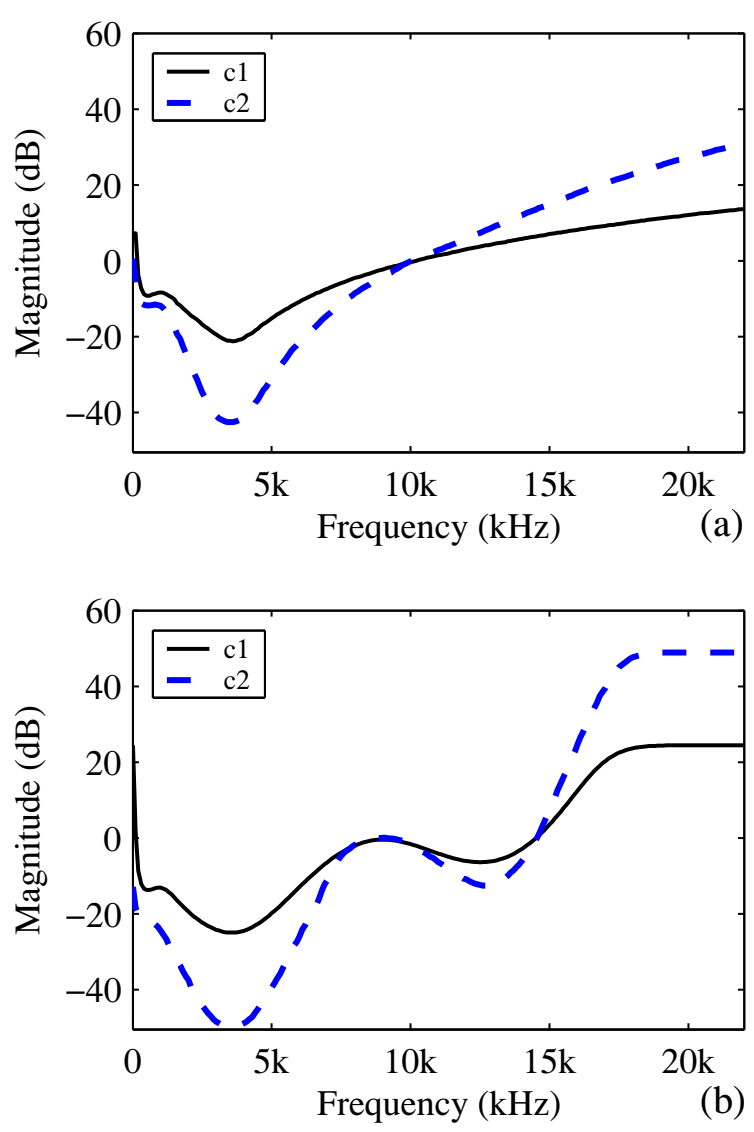

Figure 4. The design curves (modified $E$ (a) and modified $F(\mathrm{~b})$ ) (c1) and their new versions (new modified $E$ (a) and new modified $F(b))$ (c2).

\section{Listening tests}

The listening tests were performed in order to observe the influence of the obtained improvement in noise shaping filter design on noise audibility. The method of paired comparison similar to the one applied in the literature [3] was used. For that purpose, 25 subjects were involved in the tests. They aged from 20 to 26 (the mean age was 22.8) of which 15 were males and 10 were females without physical disabilities. None of them reported ear abnormalities. Before the tests, the subjects were informed about the procedure.

A pair of signals (stimuli) of duration of $3 \mathrm{~s}$ each was presented through headphones with an interval of $1 \mathrm{~s}$. The subjects assessed the degree of noise annoyance in the second signal compared to annoyance of the reference one. The reference signal was composed of $1 \mathrm{kHz}$ sinusoidal signal and unshaped white noise. The amplitudes of the reference signal components were chosen in such a way that the highest degree of annoyance was assigned to 

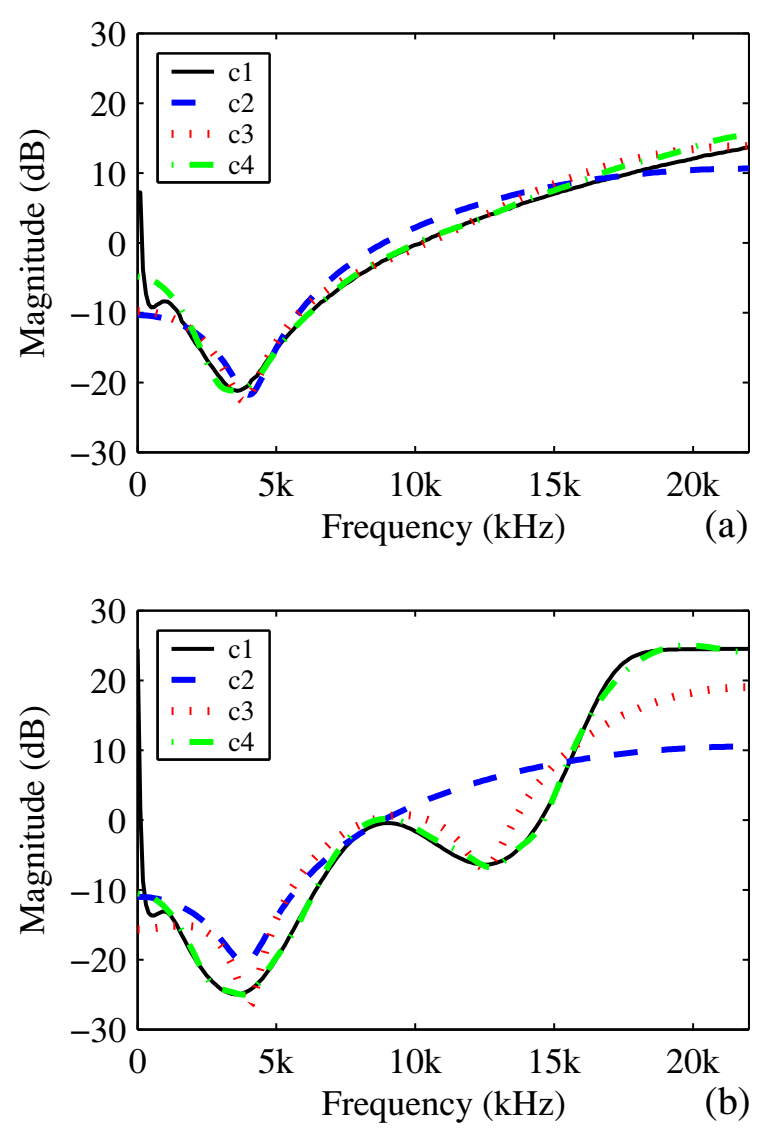

Figure 5. The target curves (c1) - modified $E$ (a), and modified $F$ (b), and PSDs of the filters of $2^{\text {nd }}(\mathrm{c} 2), 5^{\text {th }}(\mathrm{c} 3)$ and $15^{\text {th }}$ (c4) order designed by the improved procedure I using new modified $E(a)$, and new modified $F$ curve (b).

this signal (the degree 5). A number of test signals were generated by adding the unshaped or shaped noise to the $1 \mathrm{kHz}$ sinusoidal signal. Noise was shaped by applying the filters of various orders designed by both the procedure I and modified procedure I, where filtering was realized by a software module developed in Matlab.

The subjects evaluated the degree of noise annoyance of the test signal according to the five-grade scale: 5-very annoying, 4-annoying, 3-slightly annoying, 2-perceptible, but not annoying and 1-imperceptible. The subjects were allowed to take one decimal point of resolution. The averaged results of the listening tests for all 25 subjects are shown in Fig. 7.

\section{Discussion}

The PSDs of the noise shaping filters designed by the procedure $I$ using the modified $E$ and modified $F$ design

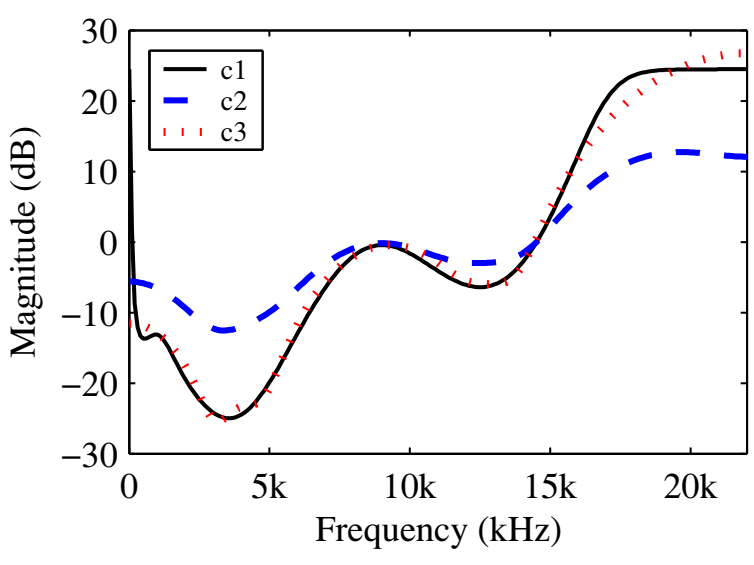

Figure 6. The target curve (modified $F(\mathrm{c} 1)$ ) and PSDs of the filters of $9^{\text {th }}$ order designed by the procedure I without (c2) and with implementation of the proposed improvement (c3).

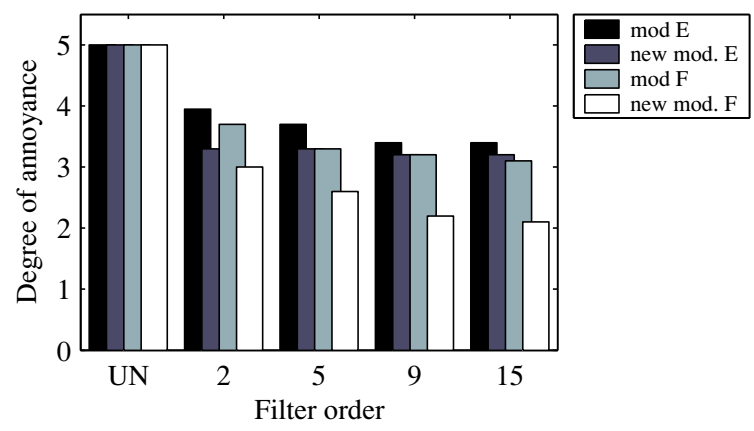

Figure 7. The averaged results of the listening tests for all 25 subjects: the degree of annoyance of noise shaped by the filters of order 2, 5, 9 and 15 designed by the procedure I using the indicated design curves (modified $E$, new modified $E$, modified $F$ and new modified $F$ ) relative to the annoyance of unshaped noise (UN).

curves (Fig. 2) show that these PSDs significantly differ from the design (target) curves. The difference is especially prominent in the frequency regions where human ear is the most sensitive. Thus, the significant difference exists around $4 \mathrm{kHz}$ for both curves and around $12 \mathrm{kHz}$ for the modified $F$ curve. Since the filters' PSDs cannot follow the design curves in the mentioned frequency regions, noise audibility is reduced to rather less extent than it could be. Increasing the filter order improves the situation to a certain extent, but only in the filters of lower orders. However, the filter order increase does not solve the problem in general, due to the fact that the differences between the obtained PSDs and design curves still remain pronounced even in the high order filters. This is confirmed by the RMS errors given in Table 1, since the errors are greater than $5 \mathrm{~dB}$ and $8 \mathrm{~dB}$ for the modified $E$ and modified $F$ curve, respectively, even in the filters of order 15 . 
Comparing the design procedure I with the procedure II (when the modified $E$ and modified $F$ design curves are used, see Fig. 2 and 3), it is observed that the procedure II gives better results, i.e. PSDs of the filters obtained by the second procedure better match the design curves. However, this is mostly valid for higher order filters. Unfortunately, higher order filters do not represent the best solution regarding the requirements that have to be fulfilled in real time applications. So, a desirable solution would be to design a filter of low order with good characteristics.

When the new modified design curves (new modified $E$ and new modified $F$ proposed here) are used for the noise shaping filter design by the procedure I, the difference between the filters' PSDs and the target curves (modified $E$ and modified $F$ ) can be significantly reduced, as shown in Fig. 5 and Table 1. The PSDs of the filters obtained with new modified $E$ curve are similar to each other and to the modified $E$ curve (target curve) independently of filter order (see Fig. 5(a)). In the filters obtained with new modified $F$ curve, there is a certain improvement with an increase in filter order (Fig. 5(b)). Thus, the PSD of the filter of higher order matches the target curve (modified $F$ curve) better. The difference between the filter's PSD and the target (modified $F$ ) curve in the frequency regions of the ears' greatest sensitivity is negligible in the filters of order 9 (see Fig. 6) and higher. Besides, it is very important that low order filters also have quite acceptable characteristics (e.g. the RMS error for the $5^{\text {th }}$ order filter given in Table 1 is reduced by about $3 \mathrm{~dB}$, and it is approximately $5 \mathrm{~dB}$ ).

Based on the results of the listening tests (Fig. 7), it can be observed that the improvement (modification) suggested here leads to a less audible shaped noise in comparison to the case where this improvement is not implemented. The improvement in noise audibility is generally smaller for the modified $E$ curve, that is, new modified $E$ curve than for the modified $F$ curve, that is, new modified $F$ curve. However, it is important that even low order filters designed by using the new modified $E$ curve provide less audible noise. In addition, it is also important that the filters designed by using the new modified $F$ curve lead to a significant improvement regarding the noise audibility (i.e. annoyance reduction of about one degree).

\section{Conclusions}

This paper analyses design of the noise shaping filters and proposes the improvement in the design procedure. The aim is to design the filters whose PSDs match the corresponding target curve as closely as possible. In order to achieve this, it is suggested that the design curves (used for the filter design) should be modified in the way to minimize the differences between the target curves (not used for the filter design) and filters' PSDs, especially in the frequency regions of the ears' greatest sensitivity. The proposed improvement can be applied in the very fast and efficient design procedure. In this way, it is possible to reduce the differences between the filters' PSDs and the target curves to almost negligible level already with $9^{\text {th }}$ order filter (the RMS error is reduced by about $5 \mathrm{~dB}$, and it is close to $3 \mathrm{~dB}$ ). Even with the low order filters $\left(2^{\text {nd }}\right.$ or $3^{\text {rd }}$ order), the results are quite acceptable (the RMS error is reduced by about $3 \mathrm{~dB}$ compared with the case when the proposed improvement is not applied), which is an important characteristic of the proposed technique. Also, the listening tests show that the proposed improvement contributes to the reduction of shaped noise audibility.

\section{Acknowledgments}

This research is partially supported by the Ministry of Science and Technological Development of Serbia through the project No. 44009. The authors would like to thank the anonymous reviewers for their constructive reviews and many helpful comments.

\section{References}

[1] Pohlmann K.C., Principles of Digital Audio, $6^{\text {th }}$ ed., McGraw-Hill Professional, USA, 2010

[2] Oomen A.W.J., Groenewegen M.E., van der Waal R.G., Veldhuis R.N.J., A variable-bit-rate buried-data channel for compact disc, J. Audio Eng. Soc., 1995, 43, 23-28

[3] Komamura M., Wide-band and wide-dynamic-range recording and reproduction of digital audio, J. Audio Eng. Soc., 1995, 43, 29-39

[4] Wannamaker R.A., Lipshitz S.P., Vanderkooy J., Wright J.N., A theory of non-subtractive dither, IEEE T Signal Proces., 2000, 48, 499-516

[5] Kadlec F., Signal processing from psychoacoustic point of view, Proceedings of $18^{\text {th }}$ ICA (April 4-9, 2004 Kyoto Japan), II-1015-II-1018

[6] Lipshitz S.P., Vanderkooy J., Wannamaker R.A., Minimally audible noise shaping, J. Audio Eng. Soc., 1991, 39, 836-852

[7] Wannamaker R.A., Psychoacoustically optimal noise shaping, J. Audio Eng. Soc., 1992, 40, 611-620

[8] Dunn C., Sandler M., Psychoacoustically optimal sigma-delta modulation, J. Audio Eng. Soc., 1997, 45, 212-223

[9] Ćirić D.G., Milošević M.A., Influence of filter order and weighting curve on shaped noise audibility, Proceed- 
ings of $3^{\text {rd }}$ TELSIKS (October 8-10, 1997 Niš Serbia), 685-688

[10] Ćirić D.G., Milošević M.A., Effects of noise shaping filters, Proceedings of INTERNOISE (August 25-27, 1997 Budapest Hungary), 1159-1162

[11] De Koning D., Verhelst W., On psychoacoustic noise shaping for audio requantization, Proceedings of IEEE ICASSP (April 6-10, 2003 Hong Kong China), 413-416
[12] Yokotani Y., Geiger R., Schuller G., Oraintara S., et al., Lossless audio coding using the IntMDCT and rounding error shaping, IEEE T Audio Speech., 2006, 14, 2201-2211

[13] Hayama A., Furihata K., Asano D.K., Yanagisawa T., Acoustic characteristics of an electrodynamic planar digital loudspeaker using noise shaping technology, J. Acoust. Soc. Am., 2005, 117, 3636-3644 